\title{
DIE BEDEUTUNG DES ABENDMAHLS FÜR DIE MENSCHENWÜRDE: EINE SÜDAFRIKANISCHE GESCHICHTE ${ }^{1}$
}

\author{
Johan Cilliers ${ }^{2}$ \\ Disziplin für Praktische Theologie und Missionswissenschaft \\ Stellenbosch Universität
}

\begin{abstract}
Abriss
In diesem Artikel wird ein kurzer Blick auf den historischen Hintergrund des berüchtigten Synode-Entscheids der ,Nederduitse Gereformeerde Kerk' im Jahre 1857 geworfen, separate Abendmahlsgottesdienste „wegen der Schwachheit mancher" zu erlauben. In Anbetracht dieser Begebenheit in der Geschichte Südafrikas und mit einer relationalen Auffassung der Menschenwürde als Ausgangspunkt werden drei Thesen aufgestellt, nämlich erstens: dass die Menschenwürde davon abhängt, den „anderen“ anzunehmen, während menschliche Entwürdigung das Resultat des Ausschließens des „anderen“ ist; zweitens: aus der Perspektive des Abendmahls wird die Menschenwürde in der Umkehr traditioneller Beziehungen verliehen; und drittens: die Menschenwürde hat sakramentale Dimensionen, während menschliche Entwürdigung anti-sakramental ist.
\end{abstract}

\section{Stichworte: Abendmahl, Apartheid, Menschenwürde}

\section{Menschliche entwürdigung in Südafrika: Eucharistischer Ursprung?}

\section{Einleitung}

Der Begriff der Menschenwürde kann innerhalb einer Vielzahl wissenschaftlicher Gebiete beschrieben werden (und wurde es) - und erstreckt sich von soziologischen, anthropologischen, juristischen, psychologischen und ökonomischen bis hin zu philosophischen, ethischen und religiösen Perspektiven, um nur ein paar zu nennen. ${ }^{3}$ In diesem Artikel

Vortrag an der Humboldt-Universität zu Berlin am 5. November 2012, als Teil einer Serie öffentlicher Vorlesungen zum Thema Religion und Menscherechte im Rahmen des Deutsch-Südafrikanischen Jahrs der Wissenschaft, mit Unterstützung durch BMBF, GER und NRF (SA), sowie der Theologischen Fakultät der Humboldt-Universität zu Berlin.

2 Professor für Homiletik und Liturgie an der Fakultät Theologie, Universität Stellenbosch, Südafrika.

3 Einige Wissenschaftler differenzieren drei Bedeutungsstränge in der Tradition des Begriffs Menschenwürde, nämlich Würde als Menschlichkeit, d.h. der gleiche Wert des Menschseins, unabhängig von gesellschaftlichem Status; Würde als gesellschaftlicher Verdienst und moralisches Format, d.h. in Bezug auf gesellschaftlichen und politischen Status und/oder Vortrefflichkeit der Tugend; und Würde als Schönheit oder „Pracht“, d.h. eine ästhetische Bedeutung, die Würde mit dem Mysterium der einzigartigen Individualität und unwiederbringlichen Beziehung des Menschen zu Gott verbindet. Cf. Johannes van der Ven, Jaco S. Dreyer, Hendrik J.C. Pieterse, Is there a God of Human Rights? The Complex Relationship between Human Rights and Religion: A South African Case \{Gibt es einen Gott der Menschenrechte? Die komplexe Beziehung zwischen Menschenrechten und Religion: Ein südafrikanischer Fall \} (Leiden/Boston: Brill, 2004), 267. Alle diese Traditionen haben in der christlichen Tradition Resonanz gefunden. vgl. R. Kendall Soulen und Linda Woodhead, “Contextualising Human Dignity” \{Contextualisierung der Menschenwürde\} In: God and Human Dignity, Hrsg. R. Kendall Soulen und Linda Woodhead (Grand Rapids: Eerdmans, 2006), 5-6. Vgl. auch die multi-disziplinären Ansätze eines Dichters, eines Rechtsexperten, eines Psychologen, eines Philosophen und 
versuche ich, diese Frage von einem theologischen und besonders von einem sakramentalen Standpunkt aus zu behandeln und ich beschränke mich auf das calvinistische Verständnis des Abendmahls ${ }^{4}$ - und weiterhin auf einen diachronischen Überblick über einen besonders schmerzlichen Teil unserer südafrikanischen Geschichte. ${ }^{5}$ Dieser kurze Abriss der frühesten ${ }^{6}$ Verständnisse und Praktiken des Abendmahls in Südafrika macht klar, dass die Apartheid „ihr Leben in der Kirche um den Tisch des Herrn“ ihren Anfang nahm. ${ }^{7}$ Der Raum, innerhalb dessen die innigste Einheit zwischen Menschen hätte existieren sollen, wurde zu dem genauen Gegenteil: ein Ort der Spaltung. ${ }^{8}$ Man könnte tatsächlich sagen,

eines Theologen in Menswaardig, red. Cas Vos und Julian Müller (Johannesburg: Orion, 1994) - in dem Jahr geschrieben, als in Südafrika die ersten demokratischen Wahlen stattfanden. Der südafrikanische Wissenschaftler, Russel Botman, spricht über zwei typische Diskurs-Stränge in Südafrika bezüglich der Menschenwürde, und zwar den der Gleichwertigkeit und Versöhnung, und setzt einen dritten dazu: die Rolle der Globalisierung. H. Russel Botman, “Covenantal anthropology: integrating three contemporary discourses of human dignity \{Anthropologie des Bundes. Integration von drei zeitgenössische Diskursen über Menschenwürde\}.” In: God and Human Dignity, Hrsg. R. Kendall Soulen und Linda Woodhead (Grand Rapids: Eerdmans, 2006), 72-86.

4 Es könnte zu Recht gesagt werden, dass die religiöse Landschaft im kolonisierten Südafrika stark vom Calvinismus beeinflusst worden war. Manche behaupten sogar, dass, wenn Calvin während der späteren Phase des Aufenthalts der französischen Hugenotten am Kap noch gelebt hätte, er es vorgezogen haben würde, unter ihnen zu leben. Pieter Coertzen, Die Hugenote van Suid-Afrika \{Die südafrikanischen Hugenotten\}1688-1988 (Kaapstad: Tafelberg, 1988), 143-152. Die Einschränkung auf das calvinistische Verständnis des Abendmahls wurde gemacht, um die Abendmahlspraktiken in Südafrika während dieser Periode im Verhältnis zu dem Verständnis des Reformators selbst zu bewerten und auszulegen, aber auch, um ideologische Kritik an diesen Praktiken zu üben. Vgl. auch Fußnote 12.

5 Natürlich könnte diese Geschichte von verschiedenen Gesichtspunkten aus erzählt, und andere Forschungsansätze gebraucht werden. Welchem Weg auch immer man folgt, der verantwortungsvolle, hermeneutische Dialog mit der Vergangenheit hat zentrale Bedeutung, denn wir haben oft die Tendenz, eine reduzierte Form der Erinnerung, ein selektives Erinnern, wenn nicht sogar absolute Amnesie anzuwenden. Einerseits sollten wir die Angreifbarkeit und Schwäche unserer Akte des Erinnerns zugeben, andererseits sollten wir auch das Potenzial des Erinnerns einbeziehen, um die Vergangenheit hermeneutisch verantwortungsvoll zu interpretieren. Vgl. Paul Ricoeur, Memory, history, forgetting \{Gedächnis, Geschichte, Vergessen \} (Chicago: University of Chicago Press, 2004), 21. Erinnerung ist alles, was wir haben, das uns mit der Vergangenheit verbindet. Aber Erinnerung kann auch auf vielfache Weise missbraucht werden, z.B., auf pathologischer und therapeutischer Ebene, auf praktischer Ebene (besonders im Sinne vom Finden und Verteidigen unserer Identität) und auf ethisch-politischer Ebene. Vgl. Robert Vosloo, "Reconfiguring ecclesial identity: In conversation with Paul Ricoeur”. \{Neugestaltung ekklesiologischer Identität: Unterhaltung mit P.R.\} Studia Historiae Ecclesiasticae. Journal of the Church History Society of Southern Africa 2007, 32 (1). 273-293.

$6 \quad$ Für diesen Teil des Vortrags beschränke ich mich auf die Jahre 1828 bis 1857. Offensichtlich ist es unmöglich, dem komplexen Gefüge der Geschichte Rechnung zu tragen. Dieses Artikel reflektiert nur einige Ereignisse, besonders solche, die sich auf die Beziehung zwischen dem Abendmahl und dem Begriff der Menschenwürde beziehen. Man könnte zu recht fragen, wo man anfangen sollte, wenn man das Feiern des Abendmahl in Südafrika behandelt. „Sollte man mit dem Synodenbeschluss 1857 in Bezug auf die ,Schwachheit mancher' beginnen, oder noch weiter zurückgehen, zu einer möglichen Abendmahlsfeier von Vasco da Gama und seinen Leuten im 15. Jahrhundert? Oder sollte man die jüngeren Entwicklungen als Ausgangspunkt nehmen, wie zum Beispiel die Zulassung von Kindern zum Abendmahl, 1998 von der Nederduitse Gereformeerde Kerk (DRC) eingeführt?“ Cas Wepener, „Immer noch „wegen der Schwachheit mancher"? A descriptive exploration of the Lord's Supper in South Africa, 1948-2002 \}.” In: Jaarboek voor liturgie-onderzoek \{Eine beschreibende Untersuchung des Abendmahls in Südafrika Jahrbuch für LiturgieUntersuchung\}18 (2002), 139.

7 Allan Boesak, Vorwort, In Apartheid is a Heresy.\{Apartheid ist Häresie\} Ed. John De Gruchy und Charles Villa-Vicencio (Cape Town: David Philip Publisher Pty Ltd, 1983), xi.

8 "Schisma ist die Spaltung des Leibes Christi. Es bedeutet Trennung am Abendmahlstisch. Es bedeutet, dass die gemeinsamen Elemente von Brot und Wein nicht gebraucht werden, um die Gemeinsamkeit des Herrn und all den Gliedern seines Leibes anzuzeigen, sondern vielmehr die Trennung der Glieder von einander und die Verweigerung von Verbundenheit sowie Gemeinschaft. Ob man würdig essen und trinken kann, wird durch 
dass das „Missverständnis von Calvins Auffassung des Abendmahls eine entscheidende Rolle in der südafrikanischen Erfahrung der Apartheid-Theologie und -Ekklesiologie und den darauf folgenden Bemühungen um die sichtbare Einheit der Kirche gespielt hat. Die Geschichte der Apartheid war auch die Geschichte des gescheiterten Abendmahls.“ 9

Diese „Geschichte des gescheiterten Abendmahls“ hört sich wie ein Kriminalroman an - ohne das glückliche Ende. Um es kurz zu machen, liste ich die drei „Argumente“ auf, die von frommen und hingebungsvollen Calvinisten gegen die gemeinsame Feier des Abendmahls im frühen, kolonialisierten Südafrika ins Feld geführt wurden.

\section{Das Argument der "religiösen" Identität}

Der erste dokumentierte Fall eines Einwands gegen die Gemeinschaft um den Tisch des Herrn geht auf 1828 zurück. Das Protokoll der Synode 1829 zeigt auf, dass ein gewisser Pfarrer ("Dominee”) J Spijker die Synode ersucht hat, ihren Standpunkt hinsichtlich der Segregation am Tisch des Herrn einzunehmen. Das war das Resultat eines Aufruhrs in seiner Gemeinde (Somerset-Hottentots-Holland - heute Somerset-West), als ein Mann namens Bentura Johannes ${ }^{10}$ in der Gemeinde getauft wurde und daraufhin zusammen mit den weißen Gemeindegliedern am Abendmahl teilnahm. Bentura wurde im Taufregister als „bastaard“ ausgewiesen, d.h. als Mischling. Sofort nach diesem Abendmahlsgeschehen protestierten gewisse Gemeindeglieder gegen die Tatsache, dass Bentura es gewagt hatte, mit „geborenen Christen“ am Abendmahl teilzunehmen. ${ }^{11}$

Dieser Ausdruck („geborene Christen“) sollte erklärt werden. Es ist interessant zu vermerken, dass die Frage nicht war, ob Bentura ein „wiedergeborener Christ“, oder so ähnlich, war, aber dass er nicht als Christ geboren wurde. Die Schlussfolgerung ist hier, dass zwischen (traditionellen, bekennenden) Christen und (früheren) Heiden unterschieden wurde. Aber die Ironie ist, dass Bentura in die Gemeinde getauft wurde, ohne dass jemand einen Einwand gehabt hätte. Deshalb wurde er als (wiedergeborener?) Christ akzeptiert. Doch war das wohl nicht gut genug. Um am Tisch des Herrn teilzuhaben (jedenfalls mit den Mitgliedern der Gemeinde, in die er getauft worden war), musste er als Christ geboren sein. Warum diese Voraussetzung?

Meiner Meinung nach war dieser Ausdruck einer von vielen, die die echten Absichten und Agenden verschleiern sollten. ${ }^{12}$ Was wirklich gemeint war, war keinesfalls, dass

andere Kriterien bestimmt, als dass Jesus seinen Leib geopfert hat. Kein Geben, sondern Besitzen wird das Kriterium des Würdigseins, und Schisma macht das Sakrament ungültig.“ Boesak, Vorwort, xii.

9 Dirkie J. Smit, “Beyond Augsburg: Calvin on the Real Presence of Christ in the Lord’s Supper” $\{$ Nach Ausgburg: Calvin über die wahre Gegenwart Christi im Abendmahl\}. Lutheran Forum 2012,46 (1) 44.

10 Vor seiner Taufe war er als Bentura Visser bekannt.

11 Für eine umfassende Debatte dieses und anderer Vorfälle, vgl. Chris Loff, "The history of a heresy” \{Geschichte einer Häresie\}. In Apartheid is a Heresy. Ed. John De Gruchy und Charles Villa-Vicencio (Cape Town: David Philip Publisher Pty Ltd, 1983), 10-23.

12 Interessant ist in diesem Zusammenhang, welche Rolle Sprache in der Gestaltung und Aufrechterhaltung zerstörerischer Ideologien spielt. Deshalb ist die Untersuchung einer Ideologie gleichzeitig auch immer eine Untersuchung davon, wie Sprache gebraucht wird um Herrschaftsverhältnisse zu sanktionieren, und es ist die Aufgabe ideologischer Kritik, auf die andern Möglichkeiten der Sprache hinzuweisen, d.h. die existierende Realität der gebrauchten Sprache als Machtinstrument zu durchbrechen, die exisiterenden Machtbeziehungen zu relativieren und neue Realitäten auf einfallsreiche Art vorzuschlagen. Einige der linguistischen modi operandi der Ideologiebildung, die der Macht-Ideologie dienen, können zum Beispiel mit einer Vielzahl linguistischer Mittel verbunden werden und einen „ewigen“ Stand der Dinge schaffen. Vgl. John T. Thompson, Studies in the Theory of Ideology \{Studien in Ideologie-Theorie\} (Cambridge: Polity Press, 1984), 131-132. 
Bentura nicht als Christ geboren wurde, sondern dass er nicht weiß war. Das Argument für diesen Ausschluss war kein geistlicher, sondern ein ethnischer; die „religiöse“ Identität, die so eifrig bewacht wurde, war nicht religiöser Art, sondern biologisch - ethnisch. Das Wort "geboren" gibt den wirklichen Vorsatz preis; "geboren" bedeutet dann genau das: ein biologischer Teil einer gewissen ethnischen In-Gruppe zu sein. Und, in diesem Sinn, wurde Bentura - ein getauftes Gemeindemitglied - disqualifiziert. ${ }^{13}$

\section{Das Argument der „legalen und moralischen“ Kategorisierung}

Zugunsten von Pfarrer Spijker muss gesagt werden, dass er nicht unbedingt der gleichen Meinung wie der Kirchenvorstand war, der die Segregation in der Gemeinde forderte; tatsächlich musste er harte Kritik für seine Einstellung, dass keine getauften Mitglieder vom Abendmahl ausgeschlossen werden dürften, über sich ergehen lassen. Die protestierenden Kirchenväter haben jedoch ihre Kampagne nicht eingestellt - sondern, als sie sahen, dass die erste Salve nicht das gewünschte Resultat brachte, änderten sie ihre Einwände und wandelten sie zu einer „moralischen“ Kategorisierung um. Die „Logik“ dieses Arguments war folgendes: Bentura hat sich der „Prostitution“ schuldig gemacht. Warum? Weil er mit der Sklavin, mit der er lebte und Kinder hatte, nicht verheiratet war. Ironischerweise war dieses Argument bei seiner Taufe nie zur Sprache gekommen, im Gegenteil, ihm wurde ein gutes Leumundszeugnis gegeben.

Aber die Ironie vertieft sich noch. Bentura hatte eine langjährige Beziehung zu der Sklavin, die seine Frau und Lebenspartnerin war, aber aus dem einfachen Grund, dass die Gesetze damals Heirat zwischen Sklaven verboten, konnte er sie nicht heiraten. So wurde das Gesetz - offensichtlich von den frommen Kirchenvätern aufrecht erhalten - als moralisches Maß gebraucht um Bentura und seine Frau zu kategorisieren. Also: wurde Bentura nicht nur aufgrund seiner ethnischen Unangemessenheit disqualifiziert, er wurde auch als moralisch unzulänglich stigmatisiert - vom Gesetz.

Dieses Argument hatte eine weitere, ironische Auswirkung. Als die Debatte über die Segregation 1829 zur Synode gebracht wurde, schlug das gleiche „Gesetz“ auf die zurück, die es gebrauchten. Damals wurde zugelassen, dass die Regierung das Recht hatte, in Kirchenangelegenheiten mitzureden, selbst bis zu dem Punkt, dass alle Entscheidungen der Synode das grüne Licht der Regierung bekommen mussten, bevor sie umgesetzt werden konnten. So repräsentierte der „Kommissaris Politiek” (Politik-Kommissar) bei allen offiziellen Kirchentreffen die Regierung, und er überprüfte, ob die Kirche sich mit „Kirchenthemen“ beschäftigte, oder nicht. Er hatte sogar die Macht, das Veto gegen Entscheidungen einzulegen und, in manchen Fällen, zu verhindern, dass ein Thema überhaupt diskutiert wurde. ${ }^{14}$

Genau das passierte bei der Synode im Jahre 1829. Die Synode erklärte sich nicht mit einem integrierten Abendmahl einverstanden, sie wurde einfach davon abgehalten, weil der „Kommissaris Politiek” der Meinung war, dass es für eine Kirchensynode schmählich sei, eine solche Diskussion überhaupt zu erwägen! Diskriminierung am Tisch des Herrn würde, jedenfalls in den Augen der damaligen Regierung, tatsächlich „gegen das Gesetz“ sein.

13 Vgl. Robert R. Vosloo, The Welcoming Table? 500-501. The Lord's Supper, Exclusion, and the Reformed Tradition, In Strangers and Pilgrims on Earth \{Der einladende Tisch? Abendmahl, Ausschließung und die reformierte Tradition\} In: Strangers and Pilgrims on Earth. Essays in Honour of Abraham van de Beek, Hrsg. E. van der Borght and P. van Geest (Leiden/Boston: Brill, 2012), 487.

14 Vgl. Loff, The History of a Heresy, 16-17. 
Ohne Frage war diese Interpretation nicht im Sinne derer, die die eucharistische Segregation verbreiteten.

\section{Das Argument der Heiligen Schrift}

Aber die Geschichte wird noch spannender. Wenn religiös-ethnische Beweggründe oder gesetzliche Bedingungen nicht genügten, dann doch sicher, was die Heilige Schrift zu dem Thema sagen würde? Es ist absolut erstaunlich zu sehen, wie viele „biblische“ Argumente gebraucht wurden, um die Menschen am Tisch des Herrn zu trennen. Einer der Kirchenältesten in Pfarrer Spijkers Gemeinde behauptete, dass Lukas 17:7-8 ein deutlicher Hinweis darauf sei, dass aufgrund der Umstände von Benturas Erbe und seinem untergeordneten Status gewisse Unterschiede am Tisch des Herrn gemacht werden könnten. ${ }^{15}$ Dieser Älteste argumentierte sogar, dass Diskriminierung keinen Schaden anrichten könne, „,weil der Erlöser selbst davon gesprochen hätte.“ ${ }^{16}$ Kein Gegenargument konnte diesen Ältesten beeinflussen. Im Gegenteil, er holte eine weitere biblische Bestätigung für die Trennung heraus: Im 1. Korintherbrief, 10:16 steht deutlich, dass das Fleischessen einen Bruder zu Fall bringen könne und deshalb solle man dann nie wieder Fleisch essen, also, wenn Benturas Anwesenheit am Tisch des Herrn bei den anderen Mitgliedern so ein Unbehagen auslösen würde, sollte er - Bentura - doch lieber wegbleiben, oder das Abendmahl getrennt feiern. Auf jeden Fall, fügte dieser Älteste hinzu, hätte Bentura nicht einmal den Anstand, dem Dankesgottesdienst nach dem Abendmahl beizuwohnen. Ein weiterer Ältester schloss sich dem bibelzitirenden Protestler an - und erinnerte Pfarrer Spijker daran, dass im 5. Buch Mose 23:2 deutlich steht, dass „kein Bastard in die Gemeinde des Herrn kommen darf, selbst bis zur zehnten Generation“, und dass Jesus nicht gekommen sei, um das Gesetz Gottes und die Propheten zu relativieren. Und so geht es mit der biblischen Grundlage der Trennung am Tisch des Herrn weiter.

Den aussagekräftigsten Gebrauch einer diesbezüglichen Bibelstelle kann man in der Entscheidung sehen, die die Synode 1857 getroffen hat. ${ }^{17}$ Damals hatte die Diskussion an Intensität zugenommen und in manchen Gemeinden wurde Segregation de facto praktiziert. Die Zeitspanne vor der Synode 1857 liefert einige fesselnde Argumente verschiedener Kirchenräte, zum Beispiel, dass, obwohl das Abendmahl nicht an verschiedenen Tagen gehalten werden solle, es problemlos am gleichen Tag zu zwei verschiedenen Zeiten gehalten werden könne. Oder: ist es nicht vorzuziehen, besondere Sitzplätze in einem separaten Teil des Kirchenraumes zu haben, meist hinten in der Kirche, für Schwarze und „Farbige“? Oder, am schlimmsten: ist es nicht vertretbar, für Weiße separate Kirchenbänke zu haben - mit Rückenlehnen - und Kirchenbänke ohne Rückenlehnen für die Heiden? In

\footnotetext{
Dieser bestimmte Absatz handelt von der Pflicht eines Dieners.

Loff, The History of a Heresy, 13.

17 Der historische Hintergrund dieser infamen Entscheidung ist relativ komplex und kann hier nicht in allen Einzelheiten erörtert werden. Kurz gefasst geht er darauf zurück, dass einige Mitglieder der Gemeinde Stockenström im Ostkap ein Gesuch eingereicht hatten, separate Abendmahlsgottesdienste halten zu können. Dieses Gesuch wurde vom Kirchenrat abgeschlagen, wurde aber vom Presbyterium Albany, unter das Stockenström fiel, wieder aufgenommen. Schließlich landete das Gesuch bei der Synode 1857. Es gab stark auseinanderlaufende Meinungen zu dieser Frage, und der Entschluss, von Reverend Andrew Murray Senior formuliert, war ein Versuch, einen Kompromiss zu finden. Jedoch hatte er die unbestreitbare Folge, die Trennung am Tisch des Herrn zu legitimieren. Eine detaillierte Übersicht dieses Hintergrunds der Synode 1857 findet sich in Christoff Pauw, Anti-apartheid theology in the Dutch Reformed Family of Churches. A depth-hermeneutical analysis \{Anti-Apartheid Theologie in der Nederlandse Gereformeerde Kirchenfamilie. Eine tiefenhermeneutische Analyse\} (Amsterdam: Vrije Universiteit, 2007), 71-76.
} 
einer Gemeinde war die Regel, dass Schwarze in der Sakristei Gottesdienst halten würden „weil in dem Kirchengebäude kein Platz sei“, aber auch daran wurde Anstoß genommen und schließlich wurden die Gottesdienste für Schwarze im Schatten eines Baumes vor der Sakristeitüre abgehalten. ${ }^{18}$

Bei der Synode 1857 versuchte man, einen Kompromiss in dieser andauernden Debatte zu erreichen und daraus ergab sich der Gebrauch der Heiligen Schrift in bemerkenswerter Weise. Der offizielle Beschluss war wie folgt:

Die Synode erachtet es wünschenswert und im Sinne der Heiligen Schrift, dass unsere heidnischen Mitglieder akzeptiert und wo immer möglich in unsere Gemeinden aufgenommen werden, aber wo diese Maßnahme durch die Schwachheit mancher der Arbeit Christi unter den Heiden im Wege stehen würde, dann sollten die Gemeinden, die unter den Heiden ins Leben gerufen wurden oder noch werden die christlichen Privilegien in separaten Gebäuden oder Institutionen genießen.

Bemerkenswert ist die Tatsache, dass dieser Beschluss in der Absicht gefasst wurde „das Werk Christi zu fördern“ - eine Art „theologischer Legitimation“ des status quo der Trennung am Tisch des Herrn. ${ }^{19}$ Schließlich wurde „die Schwachheit mancher“ das Alibi zur Rassentrennung am Tisch des Herrn, und, absolut logisch, führte es dazu, dass 1881 separate Kirchen innerhalb der sogenannten „Niederdeutsch-Reformierten Familie“ geschaffen wurden, mit der Gründung der dann Nederduitse Gereformeerde Sendingkerk (niederländisch-reformierten Missionskirche) genannten Gemeinden. ${ }^{20}$ Aber die „Logik“ dieser Trennung ging weiter und tiefer - und bot denen, die später an die Macht kamen, religiösen und „theologischen“ Treibstoff, um eine separate Gesellschaft im Namen der „freundlichen Nachbarschaftlichkeit“ zu schaffen (in den Worten eines früheren Premierministers, Hendrik Verwoerd), aber tatsächlich um die menschenunwürdige Apartheid zu etablieren, die 1948 offiziell eingeführt wurde. ${ }^{21}$

\title{
Menschliche entwürdigung in Südafrika: Drei eucharistische Thesen
}

\author{
Einleitung
}

18 Chris Loff, Dogter of Verstoteling? Kantaantekeninge by die geskiedenis van die Nederduitse Gereformeerde Sendingkerk in Suid-Afrika \{Tochter oder Verstoßene? Glossen zur Geschichte der Nederduitse Gereformeerde Sendingkerk in Suid-Afrika\} (Kaapstad: Predikante Broederkring, 1981), 22. Loff, Dogter of Verstoteling?, 22; vgl. auch Vosloo, The Welcoming Table?, 485.

20 "Der logische Schritt, separate Sitzgelegenheiten für Schwarze einzurichten, war, separate Gebäude für den Gottesdienst der schwarzen Gemeindeglieder zu bauen. Und von da an war es nur eine Frage der Zeit, bevor die separaten Gottesdienste in dem besonderen kleinen Gebäude sich zu einer separaten Gemeinde entwickelten. Und aus diesen separaten Gemeinden wurde die erste separate, oder Tochterkirche, gegründet.“ Chris Loff, "Racism, economic dependence and the Family of Dutch Reformed Churches" \{Rassismus, wirtschaftliche Abhängigkeit und die Nederlandse Gereformeerde Kirchenfamilie\} In: Conference on Economic Development and Racial Domination \{Konferenz über wirtschaftliche Entwicklung und rassistische Domination\} (Bellville: University of Western Cape, 1984), 4. Der Nederlandse Gereformeerde Missionskirche - die erste sogenannte "Tochterkirche" - folgte die Nederduitse Gereformeerde Kirche in Afrika (1910, 1932, 1951, 1952) und die Gereformeerde Kirche in Afrika (1965). Diese Kirchen dienen jeweils meist den „farbigen“, schwarzen und indischen Gemeinden. Im Jahre 1994 vereinigten sich die Nederduitse Gereformeerde Missionskirche und ein großer Teil der Nederduitse Gereformeerde Kerk und bildeten die heutige Unierte Reformierte Kirche im südlichen Afrika (URCSA), die die Belhar Confession als fundamentalen Ausdruck ihres Kampfes gegen Rassismus und die Apartheid anerkannten.

21 Im Jahre 1948 kam die Nationale Partei an die Macht und Apartheid wurde offiziell ins Landesgesetz geschrieben. 
Eine der erstaunlichen - betrüblichen - Paradoxe der oben erzählten Geschichte ist die Tatsache, dass die Anstifter für Segregation nach ihren eigenen Aussagen fest im Calvinismus verankert waren. Jedoch hatte Calvin ein eindeutiges Verständnis des Abendmahls, das in Wahrheit gegen all die oben genannten „Argumente“ für eine Trennung zum Himmel schreit. Calvins Verständnis des Abendmahls ist von manchen Calvin-Wissenschaftlern unter der Rubrik der Gegenwart Christi untersucht worden. ${ }^{22}$ Fünf Dimensionen dieser Gegenwart Christi sind identifiziert worden, nämlich erstens, die lebendige und persönliche Gegenwart Christi, durch die die Gläubigen mit Christus vereint sind, mit ihm Gemeinschaft haben und in ihn eingepflanzt sind. Zweitens die geistliche Gegenwart Christi - die entsteht durch das Wort und den Geist und darum durch den Glauben. Drittens die sakramentale Gegenwart Christi, also Seine wirkliche und wahre Gegenwart, die durch die Zeichen der Sakramente gegeben ist. Viertens die eucharistische Gegenwart Christi und die Beteiligung des Gläubigen am Sakrament, die in der sakramentalen Gegenwart wesentlich ist, und schließlich ergibt sich aus der Beteiligung des Gläubigen eine ekklesiologischen Gegenwart Christi in der Kirche als sein Leib. ${ }^{23}$

Wichtig ist es hier zu vermerken, dass die Gegenwart Christi Beziehungen impliziert zwischen dem gegenwärtigen Christus und denen, für die und mit denen Er gegenwärtig ist. Als Ausgangspunkt stelle ich fest, dass der Begriff der Menschenwürde auch relational ist und nicht nur oder sosehr eine ontologische Qualität, die irgendwie in Menschen verankert ist. ${ }^{24}$ Wir „haben“ sie nicht, sondern sie wird uns gegeben und, im Kontext der Gegenwart Christi im Abendmahl verstanden, wird sie uns durch unsere Beziehung zu Christus gegeben (persönliche Gegenwart), durch das Wort und den Geist (geistige Gegenwart), durch die Zeichen des Sakraments (sakramentale Gegenwart), aber auch durch das

22 Cf. Dirk J. Smit, “... wahrhafte Teilhaber an der wahren Substanz des Leibes und Blutes Jesu Christi...” In: Johannes Calvin ökumenisch gelesen, Hrsg. André Birmelé; Wolfgang Thönissen ( Leipzig: Bonifatius, 2012), 71-96; auch Michael Welker, Was geht beim Abendmahl vor? (Gütersloh: Gütersloher Verlagshaus, 2004), 90-102. Er sagt: “Jesus versetzt sich in seine Jünger hinein... Über den Kreis der Jünger hinaus wird der gekreuzigte und auferstandene Christus so über die Identifikation mit Brot und Wein gegenwärtig.” Welker, Was geht beim Abendmahl vor?, 97. Nach Purcell “entspringt” das Geschenk des Abendmahls “aus einem alten Versprechen; es weist auf eine eschatologische Zukunft hin, die mit Hoffnung und Verheißung gefüllt ist; und deshalb schafft es eine Gegenwart”. Michael Purcell, “This Is My Body” Which Is “For You”...Ethically Speaking. The Presence of Transcendence. \{Die Gegenwart des Transzendenz Thinking 'Sacrament' in a Postmodern Age. \{Das ist mein Leib, der für euch ist...ethisch gesprochen. Nachdenken über 'Sakrament' in der Postmoderne $\}$ Ed. Boeve, L and Ries, JC. (Leuven - Paris - Sterling, VA: Peeters, 2001), 141.

23 Smit, Beyond Augsburg, 40-43. Es scheint jedoch, als habe der calvinistische Glaube diese fünf Perspektiven der Gegenwart Christi oft aus den Augen verloren: „Christus’ lebendige und persönliche Gegenwart gegen Ideen über Christus eingetauscht; Christus' geistige Gegenwart durch rein menschliche subjektive Gedanken und Gefühle ersetzt; Christus’ sakramentale Gegenwart zu bloßer Erinnerung an Ihn reduziert; Christus’ eucharistische Gegenwart nur als menschlich-gedankliche Tätigkeit verstanden; Christus’ ekklesiologische Gegenwart in einem Interessenverband gleichgesinnter Menschen abhanden gekommen." Smit, Beyond Augsburg, 44.

24 Verschiedene Wissenschaftler haben diesen Standpunkt vertreten. Vgl. C Sherlock, The doctrine of humanity \{Die Lehre der Humanität\}(Leicester: Inter-Varsity Press, 1996), 158-160. Sherlock verficht die Meinung: „Die Bestätigung persönlicher Menschenwürde kann man nur im Licht von meiner Beziehung zu anderen verstehen, und sowohl sie, als ich, sind durch und in Christus angenommen“ (160); vgl. auch Daniël Louw, der eine pneumatologische, und deshalb innewohnende Menschenwürde postuliert. Er spricht von der Wirkung, die Stigmatisierung (eine verzerrte Beziehung) auf Menschen ausübt und die ihnen die Menschenwürde raubt. Daniël Louw, Cura Vitae. Illness and the healing of life \{Krankheit und das Heilen des Lebens \} (Wellington: Lux Verbi.BM, 2008), 426; Russel Botman spricht von der bündnishaften Dimension der Menschenwürde, die eine Beziehungen nicht nur zwischen Gott und den Menschen, sondern auch zwischen den Menschen untereinader voraussetzt. Vgl. Botman, Covenantal anthropology, 82-86. 
Teilhaben der Gläubigen am Abendmahl (eucharistische Gegenwart), sowie als Resultat der Beziehung des lebendigen Christus zu Seinem Leib, der Kirche (ekklesiologische Gegenwart).

Im Sinne dieser postulierten Verbindung zwischen der Gegenwart Christi, Beziehungen und Menschenwürde stelle ich drei (eucharistische) Thesen auf, die unter anderem auf der oben erzählten südafrikanischen Geschichte beruhen. Ich glaube, dass die historische Entstellung des Abendmahls dauerhafte Implikationen für die Menschenwürde in Südafrika hatte, weil sie unsere Beziehungen grundsätzlich beeinflusst hat. Die erste dieser Thesen ist: dass die Menschenwürde davon abhängt, den „anderen“ anzunehmen („to embrace the other"), während menschliche Entwürdigung das Resultat des Ausschließens des „,anderen “ ist.

\section{Menschenwürde zwischen exklusiver Enklave und inklusiver Annahme}

Die Synode 1857 versuchte, im Interesse „der Schwachheit“ mancher einen Kompromiss zu schließen. Diese „schwächeren“ Gläubigen suchten wahrscheinlich nach Wegen, „ihre Identität zu bestätigen und ihr Überleben zu gewährleisten. In Situationen wie dieser, die heutzutage in verschiedenen Formen mit immer größerer Häufigkeit in unserer globalisierenden und polarisiereden Welt auftreten, ist man sehr versucht, die Gnade von Gottes Annahme im Namen des Identitäts-Schützens, Sicherheits-Herstellens oder Überlebens-Garantierens zu gefährden. Zwischen diesen Versuchungen dient das richtige Austeilen des Abendmahls der Kirche als ständige Erinnerung daran, Gottes bewegende Gastfreundschaft sichtbar zu verkörpern.“25

Könnten wir behaupten, dass die Versuchung, eine Form „ethnisch-religiöser“ Identität als Argument, Überleben und Sicherheit zu schützen, heute in Südafrika immer noch lebt? Die Nederduitse Gereformeerde Kerk zum Beispiel, ist immer noch überwiegend weiß. ${ }^{26}$ Es scheint, als durchdringe das Syndrom der Enklavierung, mit seiner Tendenz auszuschließen mehr als einzuschließen, immer noch mindestens gewisse Kreise der südafrikanischen kulturellen und religiösen Landschaft. ${ }^{27}$

25 Vosloo, The Welcoming Table?, 500-501.

26 Die Entscheidung der Synode des Jahres 1857 wurde von der West-Kap Synode der Nederduitse

Gereformeerde Kerk im Oktober 2007 für ungültig erklärt und danach von der Ost-Kap Synode mit einer ähnlichen Entscheidung. Bei der West-Kap Synode nahm Allan Boesak (damals Moderator der West-Kap Synode der Unierten Reformierten Kirche) diese Entscheidung in einer ergreifenden Rede entgegen, als er unter anderem sagte: „Die Entscheidung des Jahres 1857 wurde unter dem Zeichen der Exklusivität und menschlicher Schwachheit gefasst, die Entscheidung von 2007 ist im Zeichen der Inklusivität von Gottes Annahmen getroffen worden. “ Handelinge van die Vyf en veertigste vergadering van die Sinode van die Ned Geref Kerk in Suid-Afrika (Wes-en Suid-Kaapland) \{Sitzungsprotokoll von der 4 . Versammlung der Synode der 'Nederduits Gereformeerde Kerk’ in Südafrika (West- und Süd-Kap)\}, 15-19 Oktober 2007, 116. Die tatsächliche Situation - nach 5 Jahren - ist jedoch immer noch, dass die Nederduits Gereformeerde Kerk'überwiegend weiß ist und dass die ersehnte Vereinigung der Nederduitse Gereformeerde „Kirchenfamilien“ noch nicht stattgefunden hat. Kürzlich ist ein Buch erschienen, in dem die Stimmen 18 prominenter Theologen und anderer bekannter Südafrikaner laut werden, und in dem grundsätzlich eine viel kritischere Auswertung der Belhar Confession gefordert wird, was beweist, dass dieses fundamentale Dokument in gewissen Teilen der Kirche und Gesellschaft immer noch nicht akzeptabel ist. Vgl. Belhar Geweeg \{Belhar abgewogen\}. Redakteur Piet Theron (Johannesburg: Kraal Uitgewers, 2012).

27 Wenn man dieses Syndrom der Enklavierung an Calvins Gedanken über das Annehmen des ,anderen“ misst, werden die Unterschiede mehr als offensichtlich. „Da unser Heiland uns gezeigt hat, dass das Wort „Nachbar“ den entferntesten Fremdling mit einschließt, gibt es keinen Grund, das Gebot der Liebe nur auf unseren eigenen Bekannten zu beschränken. Die gesamte Menschheit, ohne Ausnahme, muss mit dem einen Gefühl der Nächstenliebe umfangen werden: dass es keinen Unterschied gibt zwischen Griechen oder Heiden, 
Wenn man die „Afrikaner Identität“ als Beispiel nimmt, könnte man sicherlich die folgende Diagnose stellen:

Eine Enklave - wie zum Beispiel die, die sich vor und während der Apartheid um die „Afrikaner Identität“ bildete - differenziert sich von anderen Gruppen, um einen inneren Zusammenhalt zu bekommen. Eine Enklave richtet sich gegen „die anderen“, die, wiederum am Beispiel der historischen Afrikaner Identität, als „,anderes“ gesehen werden konnten: ein „anderes“ Reich (wie das britische - während der Burenkriege), „andere“ Rassen (wie es während der Apartheid zum Ausdruck kam), „andere“ Sprachen (zum Beispiel während der sogenannten „Taalbeweging“ oder „Sprachbewegung“), usw. Enklaven bedienen sich oft der Angst-Syndrome (die „schwarze Gefahr“, oder die „rote“, d.h., der Kommunismus, oder die „römische“, d.h. die „katholische Gefahr“, usw.,) und (oft extremer) Anstrengungen, die „Reinheit“ der Enklave aufrecht zu erhalten. Bei typischer Enklaven-Mentalität ist man entweder “in” oder “out”. Keine Kompromisse, keine Grauzonen - die Dinge sind schwarz und weiß. ${ }^{28}$

Es scheint fast so, als sei die Tendenz sich nach innen zurückzuziehen und die Schuld auf „andere“ zu projizieren, sogar 18 Jahre nach dem offiziellen Niedergang der Apartheid noch mit uns. Es ist, als ob der Mythos der Trennung zwischen „uns“ und „ihnen“, der so wesentlich zur Apartheid-Ideologie gehörte, zurückgekehrt ist und uns heimsucht. Die legalisierten Grenzen der Enklave mögen abgeschafft worden sein, aber das bedeutet nicht unbedingt, dass der Geist der Enklave in Südafrika nicht mehr lebt und floriert, jedenfalls innerhalb gewisser Nederduitse Gereformeerde Kerk-Realitäten. ${ }^{29}$

Der Begriff, den „anderen anzunehmen“ zeigt wiederum, dass Menschenwürde nicht nur oder so sehr eine persönliche, den Menschen irgendwie inhärente Qualität ist, sondern ein relationeller Begriff - so wie Ausschließung als eine Art „Verhältnis“ beschrieben werden könnte, das die menschliche Entwürdigung zur Folge hat. ${ }^{30}$ Das würde implizieren,

Würdigen oder Unwürdigen, Freund oder Feind; denn wir sollen die Menschen ja in Gott, und nicht an und für sich selber, ansehen. Wenn wir uns von diesem Blickpunkt abwenden, ist es nicht verwunderlich, dass wir uns in Irrtum verwickeln. Wenn wir deshalb den echten Kurs der Liebe einhalten, muss unser erster Schritt nicht sein, dass wir unsere Augen auf den Menschen richten, dessen Anblick öfter eher Hass als Liebe hervorrufen könnte, sondern auf Gott, der verlangt, dass die Liebe, die wir ihm schulden auf die ganze Menschheit verbreitet wird, sodass unser Grundprinzip immer sein muss, lass einen Menschen sein wer immer er auch sei, er muss doch geliebt werden, weil Gott geliebt wird.“ John Calvin, Institutes of the Christian Religion \{Unterweisung in der christlichen Religion\}). Ed. John T. McNeill. Übersetzt von Ford Lewis Battles. 2 Bände Library of Christian Classics (Philadelphia: Westminster Press, 1960), II/8.55. Es ist klar, dass man Calvins Gedanken in dieser Beziehung nicht einfach mit der Idee "Annahme des anderen" gleichsetzen kann, wie z.B. in Werk Miroslav Volfs (vgl. Fußnote 30). Natürlich existieren historische Brüche. Aber es bleibt eine bemerkenswerte Tatsache, dass Calvin umfänglich über die Beziehung unserer Position als Christen und denen außerhalb, den „entferntesten Fremden“ gesprochen hat. Er sagte sogar, dass sie, zusammen mit der ganzen Menschheit, „,angenommen“ werden sollten, wie in dem Zitat oben.

28 Johan Cilliers and Ian Nell, "Within the enclave: Profiling South African social and religious developments since 1994 \{Innerhalb der Enklave: Beschreibung der südafrikanischen gesellschaftlichen und religiösen Entwicklungen seit 1994\}."Verbum et Ecclesia (Skrif en Kerk) 2011; 32(1):1-7.; vgl. auch Julie Aaboe, 2007. The Other and the Construction of Cultural and Christian identity \{Der andere und der Aufbau kultureller und christlicher Identität\}: The case of the Dutch Reformed Church in Transition \{Der Fall der Nederduitse Gereformeerde Kerk im Wandel\}. Unveröffentlichte Doktorarbeit (PhD), Universität Kapstadt, 2007, 65.

29 Vgl. Johan Cilliers, "Preaching between Assimilation and Separation : Perspectives on Church and State in South African Society \{Predigen zwischen Assimilation und Trennung. Perspektiven auf Kirche und Staat in der südafrikanischen Gesellschaft\}.” In: Preaching: Does it make a Difference \{Macht es einen Unterschied\}? Hrsg. Mogens Lindhardt und Henning Thomsen. Studia Homiletica 7 (Frederiksberg: Aros Forlag, 2010 ), 73.

30 Miroslav Volf spricht von dem "Drama” des Annehmens in vier Akten, nicht außer Acht lassend, dass die Vorstellung der „Annahme“ in verschiedenen Kulturen sehr unterschiedlich gesehen werden könnte. Miroslav Volf, Exclusion \& Embrace: A Theological Exploration of Identity, Otherness, and Reconciliation 
dass jene innerhalb der Enklave ihre Menschenwürde verlieren, genauso wie jene, die ausgeschlossen werden, ihrer Menschenwürde beraubt sind. Ausschluss verursacht, und erhält, menschliche Entwürdigung - für alle Beteiligten. Im Gegenstück verweist Annahme auf einen habitus, eine Art und Weise des Umgangs miteinander, eine Möglichkeit aus der Enklave herauszutreten und den anderen in einem Akt der restorativen Würde zu suchen. Das Annehmen, im Sinne des Abendmahls, weist auf ein Hineinwachsen in die Beziehung mit dem gegenwärtigen Christus hin, aber auch mit Seinem Leib, der Kirche (Christi ekklesialer Gegenwart). Und es ist in und durch diesen Akt des Annehmens, dass Menschenwürde gegeben wird.

Das führt zu unserer zweiten These: Aus der Perspektive des Abendmahls wird die Menschenwürde in der Umkehr traditioneller Beziehungen verliehen.

\section{Menschenwürde zwischen schwacher „Stärke“ und starker „Schwäche“}

Die 1857 von der Synode getroffene Entscheidung zu Gunsten der „Schwäche mancher“ stellte in Wirklichkeit eine Form des ideologischen Selbstbetrugs dar. ${ }^{31}$ Es bezeichnete einen Akt der Maskerade - mit dem Vorsatz, die Kontrolle zu bewahren und die Macht zu erhalten. Dieses ist noch ein weiterer Paradox in der südafrikanischen Abendmahlsgeschichte: das Argument, das zugunsten der „Schwachheit“ vorgebracht wurde, drückte eigentlich eine Form der Macht aus. Man könnte zurecht fragen: wegen der Schwachheit mancher - oder der Macht?

Die Mächtigen haben sich hinter der Maske „Schwachheit“ versteckt. Diese Maske war teils aus „biblischer Korrektheit“ konstruiert, aus kanonischen „Beweisen“, dass Menschen voneinander getrennt werden sollten. Es ging auch in dem Gewand des „Gesetzes-

\{Ausgrenzung und Annehmen: Eine theologische Untersuchung von Identiät, Anderssein und Versöhnung\} (Nashville: Abingdon, 1996) 140-147. Für ihn bildet es jedoch die grundlegende Metapher für die Bewegung aus der Ausgrenzung der Enklave. Volf zeigt ein deutliches theologisches Fundament für diesen Gedanken: die Heilige Schrift der Christen bestätigen, dass Gott die Gottlosen nicht ihrer Sünde überlässt, sondern gibt von Gott selbst her um sie in die Gemeinschaft zu bringen. Die vierfache Struktur des gegenseitigen Annehmens, die Volf aufstellt, kann folgendermaßen zusammengefasst werden: Der erste Akt der Annahme ist, dass man seine Arme öffnet. Das stellt das Signal des Verlangens für den anderen dar, und ein „Zeichen, dass ich in mir Raum geschaffen habe, in den der andere hereinkommen kann und dass ich eine Bewegung aus mir selbst heraus ausgeführt habe, um den Raum des anderen betreten zu können.“ (141) Als erster Akt weist es auf einen Riss im Selbst und eine einladende Geste hin. Bezeichnenderweise spricht Volf von dem zweiten Akt der Annahme als einem des Wartens. „Das wartende Selbst kann den anderen dazu bewegen, die Bewegung zu sich selbst auszuführen, aber seine Macht, das zu tun, ist die Macht des signalisierten Verlangens, des geschaffenen Raums und geöffneter Grenze des Selbst, es ist nicht die Macht, die die Grenzen des anderen durchbricht und Erfüllung des Verlangens erzwingt.“ (142-143) Annehmen bedeutet nicht, den anderen durch die Gewalt der Macht zu ergreifen. Aber, so Volf, muss auch ein dritter Akt folgen: Das Schließen der Arme. „Das ist der Zweck der Annahme, die wirkliche Annahme, die ohne Gegenseitigkeit unmöglich ist...“ (143) Diese Gegenseitigkeit verweist auf keinen harten Griff, sondern das zarte Erfassen des Respekts. Annahme sollte nicht durch Ersticken oder Erdrücken pervertiert werden. In diesem Umschließen wird die Identität des Selbst erhalten und verwandelt, und das Anderssein des anderen wird bestätigt und respektiert. Nach Volfs Metapher muss diese Phase jedoch zu Akt vier führen, die Arme wieder zu öffnen. „Wenn das Umschließen sich nicht aufheben soll, müssen die Arme wieder offen sein.“ (144) Die Aufforderung „den anderen anzunehmen“ ist nie eine Aufforderung, den anderen im eigenen Ebenbild umzuformen. Tatsächlich öffnet sich Volfs theologisches Verständnis des Umschließens dem religiösen Pluralismus, indem es die Bedeutung verschiedener religiöser und kultureller Traditionen zur Bildung persönlicher- und Gruppenidentität aufrecht erhält. Diese Identitätsbildung darf jedoch nie wieder in eine neue Form der „Apartheid“ verfallen.

31 Boesak, Vorwort, in Apartheid is a Heresy, xii. 
gehorsams“ daher, jedoch, wie wir gesehen haben, versetzte es denen, die die Maske trugen, einen Gegenschlag.

In Wirklichkeit, wurde hier eine Doppelmaske gebraucht. Wenn Menschen sich durch Masken selbst die Macht verleihen, müssen sie andere oft $\mathrm{zu}$ gleicher Zeit mit entmachtenden Masken ausstatten, mit Masken, die keine Macht verleihen, sondern Würde unterdrücken. Die eine Maske ermächtigt mit „Würde“, die andere entmachtet - bis hin zur „Demütigung“. Dabei bleibt das wirkliche Gesicht des Mächtigen, so wie das des Entmachteten versteckt, und wahrhafte Verbindungen werden unmöglich gemacht. ${ }^{32}$

Das Abendmahl entlarvt. Die Anwesenheit Christi und die Beziehung zu Ihm bewirkt eine Umkehr der Beziehungen: die Mächtigen werden schwach, und die Schwachen stark. ${ }^{33}$ Das Abendmahl, im Sinne der sakramentalen Verkörperung des Evangeliums, bringt eine doppelte Parodie zum Ausdruck: die ihn gekreuzigt haben, parodierten ihn als „der Juden König“, d.h. als mächtig. ${ }^{34}$ Aber die Parodie kommt wie ein Bumerang zu ihnen zurück: ihre Parodie wird in Wirklichkeit durch die Wahrheit, dass Jesus mächtig ist - aber mit einer anderen Art von Macht - parodiert. $^{35}$ Die Macht des Kreuzes ist anders: paradoxerweise verleiht sie Leben dadurch, dass sie Gewalt und Kontrolle - Verletzbarkeit und anscheinende Bedeutungslosigkeit entgegensetzt. ${ }^{36}$ Diese „Macht, die Schwäche ist“, tritt allen schikanierenden Gewalten gründlich entgegen; sie steht als Herausforderung an alle Personen und Realitäten, die ihre Gewalt dazu gebrauchen, anderen Erniedrigung und Leiden aufzuerlegen. ${ }^{37}$ Diese eigenartige Macht des Kreuzes untergräbt alle Formen der Gewalt.

32 Vgl. Charles L. Campbell and Johan H. Cilliers, Preaching Fools. The Gospel as a Rhetoric of Folly \{Predigende Narren. Das Evangelium als Rhetorik der Torheit\} (Waco, Texas: Baylor University Press, 2012), 173.

33 Vgl. z.B. was Paulus im 1. Korintherbrief 1:17-25 schreibt: "Denn Christus hat mich nicht gesandt zu taufen, sondern das Evangelium zu predigen, nicht mit klugen Worten, auf dass nicht das Kreuz Christi zunichte werde. Denn das Wort vom Kreuz ist eine Torheit denen, die verloren werden; uns aber, die wir selig werden, ist’s eine Gotteskraft. Denn es steht geschrieben: "Ich will zunichte machen die Weisheit der Weisen, und den Verstand der Verständigen will ich verwerfen." Wo sind die Klugen? Wo sind die Schriftgelehrten? Wo sind die Weltweisen? Hat nicht Gott die Weisheit dieser Welt zur Torheit gemacht? Denn dieweil die Welt durch ihre Weisheit Gott in seiner Weisheit nicht erkannte, gefiel es Gott wohl, durch törichte Predigt selig zu machen die, so daran glauben. Sintemal die Juden Zeichen fordern und die Griechen nach Weisheit fragen, wir aber predigen den gekreuzigten Christus, den Juden ein Ärgernis und den Griechen eine Torheit; denen aber, die berufen sind, Juden und Griechen, predigen wir Christum, göttliche Kraft und göttliche Weisheit. Denn die göttliche Torheit ist weiser als die Menschen sind, und die göttliche Schwachheit ist stärker, als die Menschen sind.“

34 Indem er über den Einzug in Jerusalem spricht, bei dem Jesus auf einem Esel ritt, erinnert Charles Campbell uns daran: „Immerzu stellt Jesus jedoch die Macht- und Regierungsbegriffe der Welt auf den Kopf. Sein Theater ist ein wunderbares Stück politischer Parodie. Bei seinem Einzug parodiert Jesus die Gewalten der Welt und ihren Anspruch auf Ruhm und Herrschaft und stellt eine Alternative zu den Machtvorstellungen der Welt zur Schau. Er kommt nicht als einer, der seine Autorität über andere ausübt, sondern als Diener, der Herrschaft ablehnt. Er kommt nicht mit Prunk und Macht, sondern als einer, der sich mit den Armen identifiziert. Er kommt nicht als mächtiger Krieger, sondern als einer, der es ablehnt auf Gewalt zu setzen. Jesus widersteht den Machtvorstellungen der Welt, und er führt die subversive, gewaltlose Regierung Gottes mitten im täglichen Leben vor.“ Campbell and Cilliers, Preaching Fools. 25-26.

35 Vgl. Joel Marcus, “Crucifixion as Parodic Exaltation,” \{Kreuzigung als parodierende Exaltation $\}$ Journal of Biblical Literature 125 (1) (2006), 86-87.

36 L. Susan Bond, Trouble With Jesus: Women, Christology, and Preaching \{Ärger mit Jesus: Frauen, Christologie und Predigt\} (St. Louis: Chalice, 1999), 127.

37 Gayle Gerber Koontz, “The Liberation of Atonement," (Die Befreiung der Sühne) The Mennonite Quarterly Review 63 (April 1989), 183: 
Aber was passiert, wenn das Alibi der „Schwachheit mancher“ gebraucht wird? Diese paradoxe Umkehr der Beziehungen zwischen jenen mit, und jenen ohne Macht wird wiederum umgekehrt. Die Umkehrung der Umkehrung wird umgekehrt; die Parodie wird parodiert (könnten wir von einer dreifachen Parodie sprechen?). Nun wird die alte Ordnung, die alte Denkweise über Macht und Schwachheit zurückgebracht, aber unter einer Maske - um den Durst nach Macht-als-Gewalt zu kaschieren. ${ }^{38}$ In der Tat reißt diese Umkehr zurück zur alten Ordnung das Herz aus dem Sakrament, das doch von einer neuen Ordnung, einer neuen Realität spricht, in der jene, die (wirklich) schwach sind, stark sind und jene, die (wirklich) stark sind, schwach sind. Im Kontrast dazu hält die alte Ordnung an einem Verständnis und einer Praxis der Beziehungen fest, an einem Machtspiel der Domination und Unterwürfigkeit, das menschliche Entwürdigung einführt und legitimiert; es ist deshalb grundsätzlich anti-sakramental.

Das bringt uns zu unserer dritten These: Die Menschenwürde hat sakramentale Dimensionen, während menschliche Entwürdigung anti-sakramental ist.

\section{Menschenwürde zwischen verkörperndem Sakrament und entkörperlichendem Sakrileg}

Es ist eine bekannte Tatsache, dass die Apartheid in ihrem Kern immer einen „religiösen“ Charakter hatte, aber sie repräsentiert Religion, die schrecklich falsch gelaufen ist - sie könnte durchaus als entmenschlichtes „Pseudo-Evangelium“ betrachtet werden. ${ }^{39}$ Möglicherweise könnte es noch expliziter gesagt werden: Die Apartheid hat ihren Ursprung in einer sakramentalen Entstellung, einer Entsakralisierung des Abendmahls, und deshalb des Leibes Christi, und folglich des Körpers derer, die am Abendmahl teilnehmen (oder vielmehr: derer, denen die Teilnahme am Abendmahl verweigert wird). Der Leib Christi wurde fragmentiert - und das zog die Fragmentierung der Kirche, und letztendlich der südafrikanischen Gesellschaft nach sich. Es war wahrhaftig eine Ketzerei mit verheerenden gesellschaftlichen Konsequenzen. ${ }^{40}$ Man kann die traurige Ironie dieser Ketzerei nicht übersehen: einerseits sagten die Kolonisten oft, dass sie das Christentum nach Südafrika gebracht hätten, d.h., sie hätten die Gegenwart Christi, die im Abendmahl gefeiert wird, nach Südafrika gebracht; andrerseits waren es genau diese frommen calvinistischen Christen, die den Leib Christi am Tisch des Herrn abwiesen und letztlich diesen Leib zersplitterten und entstellten.

\footnotetext{
38 Solche Macht-als-Kontrolle kann die Form konzeptueller Kontrolle annehmen (sie definiert, was orthodox oder ketzerisch ist), oder verhaltensbezogene Kontrolle (sie legt fest, welche Verfahren erlaubt, oder nicht erlaubt sind), oder beziehungsbezogene Kontrolle (sie versucht, Beziehungen zu manipulieren). Vgl. Campbell and Cilliers, Preaching Fools. 56.

39 Allan Boesak, "He made us all, but...” \{Er hat uns alle geschaffen, aber... $\}$ In Apartheid is a Heresy. Ed. John De Gruchy und Charles Villa-Vicencio (Cape Town: David Philip Publisher Pty Ltd, 1983), 5-7.

$40 \quad 1987$ erklärte der Reformierte Weltbund die Apartheid als sündig und deren theologische und moralische Rechtfertigung als Ketzerei. Diese Erklärung wurde dramatisch unterstrichen, als die Delegation der schwarzen Nederduitse Gereformeerde Kerk sich weigerte, mit der Delegation der weißen Nederduitse Gereformeerde Kerk das Abendmahl zu feiern, weil sie sagten, dass es unmöglich sei, das zu tun, während der status quo in Südafrika genau das Gegenteil wäre. Damals löste diese Begebenheit enorme Kontroversen und darauffolgende Diskussionen aus. Vgl. Vosloo, The Welcoming Table?, 497. Wenn man es eine Ketzerei nennt, wird die Apartheid ,aus ihrem politischen Rahmen genommen und mitten in das Kirchenleben gepflanzt. Wenn man die Apartheid behandelt, hat man es mit dem Herzen des Evangeliums zu tun: mit dem Abendmahl.“ Allan Boesak, Vorwort, in Apartheid is a Heresy, xi.
} 
Ich glaube, dass diese (sakramentale) Entstellung und Entsakralisierung des Leibes in Südafrika immer noch weit verbreitet ist, selbst nach Jahrhunderten. Es scheint, als sei sie Teil unserer DNS und wird in einer Umkehr von Menschenwürde zur menschlichen Entwürdigung sichtbar. Man könnte sagen, dass der südafrikanische Begriff $U_{b u n t u^{41}}$, der die Menschlichkeit der Menschen in Gemeinschaft mit anderen betont (,ich bin, weil du bist") in jüngster Vergangenheit in das Phänomen Into umgewandelt worden ist. ${ }^{42}$

Das Gegenteil von Ubuntu könnte Into genannt werden, was buchstäblich „ein Ding“ bedeutet. Wenn diese Wandlung stattfindet, ist man kein Mitglied der Menschheit mehr und man wird gewissermaßen ein Ding ohne Menschlichkeit. Dann bewegt man sich von ntu (dem Stammwort für Mensch) zu Into. Ich möchte hier behaupten, dass Südafrika zur Zeit solch eine Bewegung von Ubu-ntu zu Into durchläuft, in der Menschen sich gegenseitig oft nicht als Menschen behandeln, sondern als Objekte - obwohl ich genau weiß, dass ein solches Argument kontrovers und sogar einseitig sein könnte. ${ }^{43}$

Dieses Phänomen, Mitmenschen als Into zu behandeln, ist natürlich nichts Neues: unter der Apartheid wurden verschiedene Formen der Entmenschlichung-zu-Into ausgeübt und durchaus offiziell legitimisiert. ${ }^{44}$ Aber gewisse Phänomene im heutigen Südafrika können auch von dieser Perspektive aus gesehen werden: laut Statistik hat Südafrika immer noch eine der höchsten Verbrechenszahlen der Welt. Jedes Jahr werden 31 von 100000

41 In seiner Etymologie stammt das Wort Ubuntu von Zulu- und Sotho-Versionen eines traditionell afrikanischen Aphorismus ab, der oft als „ein Mensch ist ein Mensch durch andere Menschen“ übersetzt wird. Umuntu ngumuntu ngabantu. Motho ke motho ka batho. Ubuntu ist eine Kombination von Ubu und ntuletzteres ein gemeinsamer Stamm in den meisten Sub-Sahara Afrikasprachen, hieraus ergaben sich Variationen wie shintu, muntu, Bantu, wuntu, kantu, buntu, usw. Ntu bedeutet einfach "Mensch”. Natürlich könnte Ubuntu auch romantisiert werden, und wurde es oft, oder um politische und exkludierende Positionen zu fördern, die als eine Art ächtender und populistischer Ideologie funktionierten. Es ist durchaus leicht auf den Zug des Ubuntu aufzuspringen. Leider kann er als Zauberstab betrachtet und missbraucht werden, der die Gesellschaft schmieren soll, eine Art deus ex machina um wirkliche Konflikte zu überspielen, oder ihnen auszuweichen. W. van Binsbergen, Intercultural Encounters. African and anthropological lessons towards a philosophy of interculturality \{Afrikanische und anthropologische Lektionen für eine Philosophie des Interkulturalismus\} (Münster: Lit Verlag, 2003), 428, 450. Es könnte als nützliches, aber verfrühtes Beruhigungsmittel dienen. Am schlimmsten: Er kann entstellt werden, ganz besonders auch im südafrikanischen Kontext, um eine neue Form der „Apartheid“ oder Ethnokratie oder Pigmentologie zu legitimisieren, in welcher Kultur oder Rasse oder Ethnizität neue, oder wieder alte, Grenzen zwischen der Vielfalt der Menschen zieht, die unsere Gesellschaft bildet. Mdluli ist durchaus vehement in seiner Kritik, das Unbuntu heutzutage im Dienst politischen und ideologischen Strebens missbraucht wird, wenn er sagt, dass „diese Idee von der afrikanischen bürokratischen Bourgeoisie zurückgeholt worden ist, um ihre eigene Hegemonie in den politischen Machtkämpfen zu legitimieren." P. Mdluli, "Ubuntu-Botho: Inkatha’s 'People Education’” \{Menschenschulung\} . Transformation 5, 1986, 60-77.

42 Vgl. Johan Cilliers, "In search of Meaning between Ubuntu and Into: Perspectives on Preaching in Postapartheid South Africa. \{Auf der Suche nach Sinn zwischen Ubuntu und Into: Perspektiven rund um das Predigen im post-Apartheid Südafrika\}.” In: Preaching: Does it make a Difference Macht es einen Unterschied\}? Hrsg. Mogens Lindhardt und Henning Thomsen. Studia Homiletica 7 (Frederiksberg: Aros Forlag, 2010), 77-87.

43 Es könnte sogar als typisch westliche Konstruktion oder Projektion ausgelegt werden.

44 Unser relativ friedlicher politischer Wandel 1994, der von vielen als Wunder angesehen wird, könnte u.a. dem afrikanischen Gefühl des Ubuntu zugeschrieben werden. Dies bedeutete das Ende der Zeiten, in der Menschen ihrer Würde beraubt wurden und ubulwane (tierisches Verhalten) gebraucht wurde, um die Apartheidsgesetze aufrecht zu erhalten. Nach Maphisa ist “die Umwandlung eines Apartheid-Südafrika zu einer Demokratie eine Wiederentdeckung von Ubuntu.” S Maphisa, Man in constant search of Ubuntu: a dramatist's obsession \{Der Mensch auf der ständigen Suchen nach Ubuntu: Besessenheit eines Dramatikers\} (Pietermaritzburg: University of Natal, AIDSA, 1994), 8. Die Stimmung von Ubuntu hat uns zweifellos geholfen, ganz besonders auf dem Gebiet der Versöhnung. Kurz gesagt: ohne Ubuntu gäbe es wahrscheinlich kein „neues Südafrika“. Van Binsbergen, Intercultural Encounters \{Interkulturelle Begegnungen\}, 440. 
Menschen in Südafrika ermordet (15 609 im letzten Jahr), verglichen mit durchschnittlich sieben in 100000 weltweit - um nur einen Aspekt der Kriminalität in Südafrika zu nennen. ${ }^{45}$ Aber nicht nur einzelne Morde sind, worum es hier geht. Immer öfter konfrontieren Gemeinschaften die, welche die Macht haben - der Konflikt vor kurzem (am 16. August 2012) zwischen Polizei und streikenden Minenarbeitern an der Lonmin Mine im Distrikt Marikana ist ein blutiges Beispiel hiervon, und hatte den Tod von 36 Minenarbeitern, 2 Polizisten, vier unidentifizierten Menschen und 78 Verletzten zur Folge und schickte Schockwellen um die ganze Welt. In den Medien wurde dieses Massaker sogar mit dem Massaker von Sharpeville 1960 verglichen, einem der dunkelsten Tage der Apartheid. Es veranlasste die Frage: konnte so ein Ereignis wirklich in dem sogenannten neuen, demokratischen Südafrika stattfinden?

Im Licht unserer Geschichte jedoch treten solche und ähnliche Begebenheiten keineswegs unerwartet auf. Wenn Menschen als nicht-menschliche Objekte gesehen werden, ist es leicht, sie zu ermorden; wenn sie als wegwerfbare Objekte beurteilt werden, ist es leicht, sie zu erschießen. Wenn man den sakramentalen Ursprung der Apartheid vor Augen hat, könnten wir sagen, dass jeder neue Übergriff, den „wir“ gegen „sie“ verüben, jeder Schlag, der „den anderen“ trifft, grundsätzlich wieder ein weiteres sakramentales Sakrileg darstellt, wieder eine weitere eucharistische Ketzerei. ${ }^{46}$ Das Heilige des Sakrament des Leibes bildet dann zwischen dem Täter und den Opfer keinen Schutz mehr. Dann wird der Leib nicht mehr als sakramentaler Träger des Geistes betrachtet, sondern wird verdinglicht und instrumentalisiert, als Into gesehen, ein Ding. ${ }^{47}$

45 Nach am 20. September 2012 von Nathi Mthethwa, Polizeiminister, im Parlament vorgelegten Statistiken.

46 Zu wiederholen: Die, die Trennung am Tisch des Herrn vorantrieben, waren treue Calvinisten, aber wenn man diese Entheiligung menschlichen Lebens und des menschlichen Körpers mit Calvins Überlegungen über den Wert (die Würde) des Lebens vergleicht, wird es sehr deutlich, dass das Verständnis des Reformators von der Einheit des Lebens total anders war, und zwar genau entgegengesetzt, z.B., wenn er sagt: „Da der Herr das ganze Menschengeschlecht mit einer Art Einheit verbunden hat, sollte die Sicherheit aller als jedem anvertraut betrachtet werden. Im Allgemeinen ist deshalb alle Gewalttätigkeit und Ungerechtigkeit, und jeder Schaden, den der Leib unseres Nachbarn erleidet, verboten. Folglich ist es uns geboten treu zu tun was in uns liegt um das Leben unseres Nachbarn zu schützen, zu fördern was immer seiner Ruhe zuträglich ist, wachsam zu sein im Abwehren von Unheil, und, wenn Gefahr droht, bei ihrer Beseitigung Beistand zu leisten...Die Menschheit ist beides, Gottes Ebenbild und unser Fleisch und Blut. Weswegen, wenn wir das Ebenbild Gottes nicht missachten wollen, müssen wir die Menschen heilig halten - wenn wir uns nicht unserer Menschlichkeit entledigen wollen, müssen wir unser eigenes Fleisch wertschätzen.“ Calvin, Institutions, II/8.39,40.

47 Der Begriff des Sakraments des Leibes ist von vielen Wissenschaftlern vorgebracht worden. Innerhalb der reformiert-calvinistischen Tradition kann man z.B. die Wurzeln dieses sakramentalen Verständnisses des Lebens in den Werken des namhaften niederländischen Theologen, Arnold van Ruler, finden. Im Kontext dieser „relativ unabhängigen Pneumatologie“, sprach er oft von der Tatsache, dass der Geist im vollsten Sinn des Wortes in humanum eingedrungen ist, und dass eine echte Vermengung ("vermenging”) dieser Realitäten stattgefunden und durch dieses inhabitatio Spiritus Sancti der Menschheit eine neue Würde erteilt hat. Vgl. Arnold van Ruler, Theologisch Werk, Teil VI (Nijkerk: Callenbach, 1973), 21.; In einem Kommentar zu der Pneumatologie Van Rulers sagt Jac Rebel das Folgende: „Nicht nur Mensch und Kirche, aber auch Natur, Kultur, Geschichte und Regierungen sind Gebilde des Geistes.“ Jac J. Rebel, Pastoraat in Peneumatologisch Perspektief. \{Hirtenamt aus einer pneumatologischen Perspektive. Eine theologische Verantwortlichkeit aus dem Denken von A.A. van Ruler\} (Kampen: Kok, 1981), 363. Ein anderer berühmter Theologe aus der niederländischen Tradition, der eine „sakramentale Theologie“ entwickelte, war natürlich Gerardus van der Leeuw, obwohl sein Ansatz zu dem von Van Ruler sehr unterschiedlich war. Vgl. Gerardus van der Leeuw, Sacramentstheologie \{Sakramentstheologie\} (Nijkerk: Callenbach, 1949). Von seelsorgerlicher Perspektive aus gesprochen, hat Daniël Louw den Begriff von Sakramentalität in einem afrikanischen Kontext entwickelt. Vgl. Louw, Cura Vitae, 88-89. Jacques Sarano, der über den Leib als “Zeichen oder Sakrament des Geistes”, fasst den Begriff des Sakraments des Leibes treffend zusammen: „Kurz gefasst, ist der Leib keine Region der Person; er ist das wirkliche „Gesicht“ der Person, in dem man die Wünsche der Person ablesen kann. Der Leib ist nicht einfach das, wodurch der Geist eine einzigartige Person wird; vielmehr ist es durch den Leib, dass die 
Ich glaube, dass die größte Tragik der südafrikanischen Gesellschaft eine sakramentale bleibt, selbst wenn jene, die diese Tragödien verursachen, keine Ahnung davon haben, was sie eigentlich tun. Im Wesentlichen repräsentiert Kriminalität eine Form der AntiSakramentalität, Mord stellt einen anti-sakramentalen Akt dar, den schlimmsten Ausdruck zerrütteter Beziehungen, d.h. von menschlicher Entwürdigung.

\section{Schlussbemerkungen}

Es ist klar, dass die südafrikanische Geschichte des Abendmahls in ihrem historischen Kontext verstanden werden muss, und sie darf nicht überintepretiert werden, aber es ist auch klar, dass diese Geschichte eine wesentliche Rolle dabei gespielt hat, nicht nur separate Kirchen, sondern schließlich auch die Trennung verschiedener Gruppen in Südafrika zu etablieren. Die symbolische und emotionale Bedeutung der Unfähigkeit der Menschen, in diesem intimsten Raum zur Einheit Verbundenheit zu schaffen, sucht uns immer noch heim. Eine Frage bleibt offen: kann dieses negative Erbe umgekehrt werden?

Mindestens das muss gesagt werden: wenn die Zweckentfremdung des Abendmahls die Macht hatte, Menschen so grundsätzlich von einander zu trennen, wer weiß welche Macht das Abendmahl haben könnte, das gegenseitige Annehmen zu fördern? Wer weiß, welchen sakramentalen Raum das öffnen könnte - wider das gesellschaftliche Syndrom der exklusivistischen Enklave? ${ }^{48}$

Natürlich können sogar Fragen wie diese von vielen als Wunsch- und unpraktisches Denken gesehen werden, aber man stelle sich vor, welch symbolische Aussage es für alle Südafrikaner haben könnte, wenn wenigstens die Kirchen ihre Einheit innerhalb des Abendmahls-Raumes demonstrieren könnten. Es würde bedeuten, dass die verschiedenen Kirchen (Konfessionen) Grenzen überschreiten müssen, um angenommen zu werden und den anderen anzunehmen. Wir müssen uns über den Konfessionalismus hinaus bewegen, wenn wir erhoffen, irgendeine Bedeutung innerhalb der Gesellschaft zu haben. Wir müssen das Thema des hermeneutischen Raumes der ökumenischen Kirche wieder aufgreifen, um die gesellschaftlichen Übel in unserem Land anzugehen.

Wir müssen zusammen Brot brechen, und zusammen Wein trinken.

Unmöglich?

Wahrscheinlich.

Aber dennoch...

Person seinem Geist Zeuge ist, zu dem Geist. Es ist durch meinen Leib, dass ich dieses Zeugnis des Heiligen Geistes annehme oder ablehne; von diesem Ablehnen geht das Leib-Objekt hervor, der Leib-Überrest, der nicht-angenommene Leib. Der Körper ist der Dolmetscher, der Übersetzer, der Offenbarer, der Katalysator, der Sprecher, der Bote, der verkündigt - kurz, der Leib ist das Symbol meiner Wahl. Er ist der Vermittler des Geistes.“ Jacques Sarano, The meaning of the Body \{Bedeutung des Leibes\}. Übersetzt von James H. Farley (Philadelphia: Westminster Press, 1960), 117.

48 Der Begriff eines solchen heilenden, versöhnenden Raumes in der südafrikanischen Gesellschaft ist freilich komplex. Vgl. meine Diskussionen in Johan Cilliers, "Religious and cultural transformations and the challenges for the churches - A South African Perspective \{Religiöser und kultureller Wandel und die Herausforderungen an die Kirche - eine südafrikanische Perspektive\}.” Praktiese Teologie in Suid-Afrika 2007, 22 (2):1-19; auch Johan Cilliers, "Creating space within the dynamics of interculturality: the impact of religious and cultural transformations in post-apartheid South Africa \{Errichtung des Raumen innerhalb der Dynamik der Interkulturalität: Die Auswirkungen von religiösem und kulturellem Wandel im post-Apartheid Südafrika\}.” Wilhelm Gräb, Lars Charbonnier (Hrsg.) Secularization Theories, Religious Identity and Practical Theology. Proceedings of the IAPT Conference Berlin 2007. \{Sekularisationstheorien, religiöse Identität und Praktische Theologie. Protokoll der IAPT-Konferenz Berlin 2007\} Reihe: International Practical Theology Bd. 7 (Berlin: Transaction Publishers, 2009), 260-270. 